\title{
BASC-3 Response Pattern Index
}

National Cancer Institute

\section{Source}

National Cancer Institute. BASC-3 Response Pattern Index. NCI Thesaurus. Code C159861.

Detects two types of repeat patterns: repeated and cylindrical response patterning. 